\title{
La Participación de las mujeres africanas en la Educación Superior
}

\author{
Hernández Hernández, Virginia María \\ Universidad de La Laguna, Santa Cruz de Tenerife, España \\ alu0100889888@ull.edu.es \\ Ramos Rodríguez, Laura \\ Universidad de La Laguna, Santa Cruz de Tenerife, España \\ alu0100880878@ull.edu.es \\ González Pérez, Inmaculada \\ Universidad de La Laguna, Santa Cruz de Tenerife, España \\ mifonpe@ull.edu.es
}

\begin{abstract}
Resumen
El informe que se desarrolla a continuación presenta un análisis comparativo sobre la situación de las mujeres africanas en la Educación Superior en los países de Cabo Verde, Marruecos y Mauritania. Se parte de un estudio general de la situación de la mujer en África, así como una investigación sobre el contexto político, social y económico de los países seleccionados. Posteriormente, se ha realizado una recogida de datos e información vinculada a las siguientes categorías: Indicadores Educativos, Titulaciones escogidas en función del sexo y Profesorado Universitario. De esta manera, se han utilizado fuentes primarias y secundarias de carácter cualitativo y descriptivo, así como datos cuantitativos, que han permitido realizar el análisis comparativo necesario para el desarrollo de esta investigación. Dichos datos han permitido conocer las semejanzas y diferencias de género en educación existentes en los países nombrados y el grado de inclusión actual de la mujer en la Educación Superior. Asimismo, se incluirá una propuesta de mejora basada en las debilidades y fortalezas encontradas.
\end{abstract}

\section{Abstract}

This report is a comparative analysis of the situation of African women in Higher Education in the countries of Cape Verde, Morocco and Mauritania. It begins with a general study about the situation of women in Africa, as well as an investigation on the political, social and economic context of the selected countries. Afterwards, a data and information collection was carried out about the following categories: Educational Indicators, Degrees chosen depending on the sex and University Teaching Staff. In a certain way a qualitative and descriptive source have been used, as well as quantitative data which have allowed the comparative analysis required for the development of this research. These data have allowed to know the similarities and differences of gender in education and the inclusion of women in Higher Education. Likewise, this report included an improvement proposal based on the weaknesses and strengths founded.

Palabras clave: Educación Superior, Mujer, Cabo Verde, Mauritania, Marruecos.

Keywords: Higher Education, Woman, Cape Verde, Mauritania, Morocco.

\section{INTRODUCCIÓN}

El tema central elegido para el desarrollo de esta investigación ha sido la participación de las mujeres africanas en la Educación Superior en Cabo Verde Mauritania y Marruecos. Estos países han sido elegidos ya que se encuentran situados en un continente en el que durante muchos años ha predominado la exclusión de la mujer en todos sus ámbitos. Aunque en los últimos años la situación de las mismas ha cambiado, y a pesar de que todavía quede mucho por hacer, es esencial establecer cuál es su situación en la educación, concretamente en el ámbito universitario. Todo ello porque la educación supone para muchas de ellas una vía de escape para salir del mundo rural en el que viven con sus familias, poder conseguir un trabajo digno, poder ser autosuficientes y autónomas en sus decisiones y les permite poder luchar por sus derechos y exigencias.

De esta manera, el objetivo principal de esta comunicación es conocer la realidad de las mujeres caboverdianas, mauritanas y marroquís en la Educación Superior para poder contrastar el discurso social existente en la actualidad referida a la exclusión de las mismas. Para ello, se han establecido 
una serie de hipótesis que serán rechazadas o aceptadas en función del análisis de la información y los datos obtenidos. Asimismo se incluirá una propuesta de mejora basada en las diferentes realidades sociales, económicas y políticas de los países que ocupan esta comunicación.

\section{OBJETIVOS}

El objetivo general es conocer la situación de las mujeres caboverdianas, marroquís, mauritanas y malíes en la Educación Superior. Para ello, se han establecido una serie de objetivos específicos:

- Analizar los indicadores educativos en Cabo Verde, Mauritania y Marruecos.

- Identificar las titulaciones escogidas en función del sexo en Cabo Verde, Mauritania y Marruecos.

- Indicar la cantidad de profesores y profesoras en las universidades públicas de Cabo Verde, Mauritania y Marruecos.

\section{MARCO TEÓRICO}

En todos los países seleccionados, como en la mayoría de los países del mundo, sigue existiendo una cultura patriarcal que se trasmite de generación en generación debido a los pensamientos, valores y normas sociales que dejan a la mujer en una posición de inferioridad con respecto al hombre. De esta manera, la violencia doméstica, el matrimonio infantil, la poligamia y la mutilación genital son problemas comunes en todos los países, siendo factores que impiden que las niñas puedan acceder a la educación, concretamente a la Educación Superior. Además, esta cultura patriarcal sigue vigente porque existen leyes que la apoyan. Por ejemplo en Marruecos, el Código de la Familia permite el matrimonio infantil y la poligamia (CNDH, 2015).

Otro problema común es la feminización de la pobreza, es decir, las mujeres y niñas que viven en zonas rurales tienden a ser más vulnerables que el resto. Su situación de pobreza afecta al acceso a los distintos niveles educativos y sobre todo a la Educación Superior. De esta manera, las mujeres se han visto privadas del acceso igualitario a las oportunidades económicas, desarrollo humano, acceso a instituciones y leyes que protejan sus derechos, y empeorando la situación de pobreza de las mujeres (Lopes da Silva, 2016a).

Además, existen numerosas restricciones tanto a nivel local como global que dificultan el acceso de la mujer a la participación política y a los puestos de liderazgo. Sin embargo, Cabo Verde contiene el mayor número de mujeres en cargos ministeriales en África, de los cuáles en 2016 de los 17 ministros del gobierno de Cabo Verde, 9 eran mujeres (IPS, 2016).

Finalmente, en la mayoría de estos países la mejora del nivel educativo de las mujeres no es sinónimo de un mayor acceso al mercado de trabajo y al empleo de calidad. Por ello, son muchas las mujeres que no continúan estudios de Máster y Doctorado ya que estos estudios no son reconocidos como sí ocurre con los hombres (Lopes da Silva, 2016b).

\section{METODOLOGÍA}

Para llevar a cabo esta investigación se ha utilizado un método comparativo en el que se ha una realizado una comparación de la situación de las mujeres caboverdianas, mauritanas y marroquís en la Educación Superior. Para poder desarrollar la metodología indicada se ha seleccionado un enfoque por aproximación de problemas, utilizando los problemas concretos hallados en la Educación Superior. Además, se ha considerado que el nivel de aplicación más adecuado para la realización de esta investigación es el prospectivo ya que este enfoque permite establecer, de manera aproximada, lo que podrá ocurrir o no en el futuro si se sigue manteniendo determinadas tendencias.

Asimismo, los recursos metodológicos utilizados han sido el análisis de contenido y análisis estadísticos, haciendo uso de una serie de fuentes de información primarias (como UNESCO, ONU MUJERES, Instituto de Estadística de la UNESCO, UNICEF), secundarias (revistas académicas como la 
Revista de Educación, Revista de Educación Comparada, Oficina de Información Diplomática) y terciarias (como Convención sobre la Eliminación de Todas las Formas de Discriminación contra la Mujer (CEDAW), Comisión Nacional de Derechos Humanos de Marruecos (CNDH)), siendo fundamentales para el desarrollo de la investigación.

\section{HIPÓTESIS Y CATEGORÍAS}

\subsection{Hipótesis comparativas}

- La tasa de matriculación de mujeres en la Educación Superior es mayor en Marruecos que en Cabo Verde y Mauritania.

- La tasa de graduación de mujeres en la Educación Superior es mayor en Cabo Verde que en Marruecos y Mauritania.

- La tasa de repetición y deserción de mujeres en Mauritania y Marruecos es mayor que en Cabo Verde.

- En Cabo Verde, Mauritania y Marruecos los hombres tienden a matricularse en titulaciones relacionadas con ingenierías y las mujeres en titulaciones relacionadas con la educación y el cuidado.

- El número de mujeres y hombres profesores en las universidades de Cabo Verde, Mauritania y Marruecos es igual en dichos países.

\subsection{Categorías clasificatorias.}

- Indicadores educativos:

a. Tasa de matriculación.

b. Tasa de repetición.

c. Tasa de deserción.

- Titulaciones escogidas en función del sexo:

a. Educación.

b. Ciencias.

c. Artes y Humanidades.

d. Administración de Empresas y Derecho.

e. Salud y Bienestar.

- Profesorado universitario:

a. Cantidad de profesores y profesoras.

b. Responsabilidades y cargos.

\section{ANÁLISIS Y CONCLUSIONES COMPARATIVAS}

A continuación se procederá al análisis de información y datos, estableciendo si se aceptan o se rechazan las hipótesis planteadas en un principio. La primera hipótesis, que se incluye en la categoría de Indicadores Educativos, establecía que la tasa de matriculación de mujeres en la educación superior es mayor en Marruecos que en Cabo Verde y Mauritania.

Para establecer si era cierta o no, en primer lugar se atendió al total de personas matriculadas en los niveles de grado, máster y doctorado y su distribución por sexos en cada país. Esto permitió establecer que Cabo Verde es el único país en el que el número de mujeres matriculadas en el nivel de grado fue superior al de los hombres en 2016. De hecho, un 60,1\% de mujeres se matricularon en dicho 
año. En los niveles de máster y doctorado, la situación cambia, y el número de hombres matriculados supera al de las mujeres en todos los países. La desigualdad se hace más notable en Mauritania donde los porcentajes son muy inferiores al de los hombres. De hecho, en el nivel de Máster las mujeres mauritanas representan el $28 \%$ de las personas matriculadas en 2016. (UIS, 2016a).

En segundo lugar, se realizó una comparación entre el número de mujeres matriculadas en cada país. Para ello, se tuvo en consideración el número de mujeres que se encontraban en edad de cursar la Educación Superior en cada país en 2016. Esto permitió establecer que Cabo Verde es el país en el que se matricularon más mujeres en el nivel de grado y, por lo tanto, la hipótesis se rechaza en este nivel. Sin embargo, en los niveles de máster y doctorado, el número de mujeres que se matricularon en comparación con las que se encontraban en edad de estudiar es prácticamente insignificante. No obstante, en ambos niveles educativos se matricularon más mujeres marroquís que en el resto de países y, por lo tanto, la hipótesis se acepta en los niveles de posgrado.

En cuanto a la segunda hipótesis, incluida en la categoría de Indicadores Educativos, establecía que la tasa de graduación de mujeres en la Educación Superior es mayor en Cabo Verde que en Marruecos y Mauritania. En el nivel de grado de nuevo destaca Cabo Verde, ya que se graduaron en 2016383 mujeres más que hombres (UIS, 2016b). Esto es bastante significativo porque no existen prácticamente diferencias en el número de hombres y mujeres en edad de cursar la educación superior en 2016 en dicho país. Por lo tanto, el hecho de que haya más mujeres graduadas no se justifica por una cuestión demográfica.

Sin embargo, en los niveles de posgrado la situación es inversa. En el nivel de máster se graduaron más hombres que mujeres en 2016 en todos los países. La mayor desigualdad se encuentra en Mauritania, donde se graduaron 90 hombres más que mujeres (UIS, 2016b). En cuanto al nivel de doctorado, no se ha podido establecer una comparación porque los porcentajes eran nulos o insignificantes.

De esta manera, una vez analizado el número de mujeres graduadas, se pudo establecer que en el nivel de grado la hipótesis es aceptada porque en Cabo Verde el porcentaje de mujeres graduadas es mayor que en el resto de países (2,9\%), aunque las diferencias son escasas con Marruecos $(2,59 \%)$ (UIS, 2016b). Sin embargo, se rechaza en los niveles de máster y doctorado porque se graduaron más mujeres en Marruecos que en Mauritania y Cabo Verde.

La hipótesis 3 establecía que la tasa de repetición y deserción de mujeres en Mauritania y Marruecos es mayor que en Cabo Verde. Esta hipótesis no pudo ser rechazada ni aceptada porque no existen datos cuantitativos referentes a la Educación Superior. Sin embargo, sí existen algunos estudios que reflejan que Marruecos logró reducir sus tasas de repetición gracias a la Reforma LMD (realizada con el objetivo de adaptarse al sistema europeo). Asimismo, el Ministerio de Educación de Marruecos ha elaborado proyectos que incorporan medidas contra la repetición y el abandono de las niñas, pero sobre todo se centran en la enseñanza primaria y secundaria, es decir, ninguno de ellos recoge medidas específicas para evitar que las mujeres repitieran o abandonaran sus estudios universitarios (UNESCO, 2017a).

En cuanto a Cabo Verde, también existe un estudio realizado por el Banco Mundial (2012) en el que se expone que las principales causas que obligan a una mujer a abandonar sus estudios son el no poder combinar el trabajo doméstico y el cuidado de sus hijos e hijas con los estudios, la falta de apoyo de los maridos, la distancia entre su casa y la universidad o por cuestiones económicas.

Otra categoría seleccionada para la realización de esta investigación se denomina «Titulaciones escogidas en función del sexo». Para ello, se ha planteado la hipótesis 4 de esta investigación: En Cabo Verde, Mauritania y Marruecos los hombres tienden a graduarse en titulaciones relacionadas con ingenierías y las mujeres en titulaciones relacionadas con la educación y el cuidado.

Para la realización esta investigación, se han seleccionado los datos encontrados en el Instituto de Estadística de la UNESCO sobre los graduados y graduadas en cinco campos de educación diferentes en el año 2016 de Cabo Verde, Mauritania y Marruecos. Esos campos de educación seleccionados son: «Educación»; "Ciencias»; «Artes y Humanidades»; Administración de Empresas y Derecho»; y Salud y Bienestar».

Tras realizar una comparación entre los porcentajes de hombres y mujeres graduados, se han seleccionado los mayores porcentajes por campo de educación en cada uno de los países. De esta manera, se ha podido observar cómo la mayor cantidad de hombres y mujeres se graduaron en el mismo campo de educación en dichos países. Es decir, en Cabo Verde el mayor porcentaje de hombres y mujeres fueron graduados en Administración de Empresas y Derecho; en Mauritania, en Artes y Humanidades; y en Ma- 
rruecos, en ciencias (UIS, 2016c). Esto llama la atención porque tanto hombres como mujeres en cada país se decanta por el mismo campo de estudio y esto puede deberse a que son carreras que suelen tener un mayor prestigio o demanda en dicho países. De este modo, se rechaza la hipótesis 4 ya que las mujeres no tienden a graduarse en carreras vinculadas a la Salud y Bienestar y Educación, sino que ha aumentado el número de graduadas en Ciencias y en Administración de Empresas y Derecho.

Por otro lado, también se ha seleccionado otra categoría para el desarrollo de la investigación denominada «Profesorado universitario». Para ello, se ha elaborado la hipótesis 5: El número de mujeres y hombres profesores en las universidades de Cabo Verde, Mauritania y Marruecos es igual en dichos países.

Tras realizar un análisis de los datos encontrados sobre la cantidad de profesores y profesoras en las universidades de dicho países en los años 2009 y 2014, se puede indicar que a pesar de que aumenta el número de mujeres y de hombres entre ambos años (excepto en Marruecos que solo hay datos del 2009), predomina una mayor cantidad de profesores que de profesoras. De esta manera, las mayores diferencias encontradas son en Marruecos en el año 2009 (8.570 hombres más que mujeres) (UIS, 2009) (UIS, 2014).

Asimismo, se ha realizado una investigación sobre las responsabilidades y cargos de cada una de las universidades públicas de los países seleccionados. Los resultados de dicha investigación demuestran que solo hay mujeres en los altos cargos en la única universidad pública de Cabo Verde (rectora y vicerrectora). Mientras que en las nueve universidades de Marruecos de las que se han encontrado datos y en la de Mauritania no hay mujeres en los altos cargos (UAE, s.f.) (UCA, s.f.) (UCD, s.f.) (UNICV, s.f.) (UH1, s.f.) (UNIVH2, s.f.) (UIZ, s.f.) (UMP, s.f.) (USMS, s.f.) (USMBA, s.f.). Por lo tanto, se debe rechazar la hipótesis plateada porque la cantidad de profesores y de profesoras en las universidades no es igualitaria.

\subsection{Tabla de yuxtaposición de datos}

\begin{tabular}{|c|c|c|c|c|}
\hline Categoría & Subcategorías & Cabo Verde & Mauritania & Marruecos \\
\hline \multirow{3}{*}{ 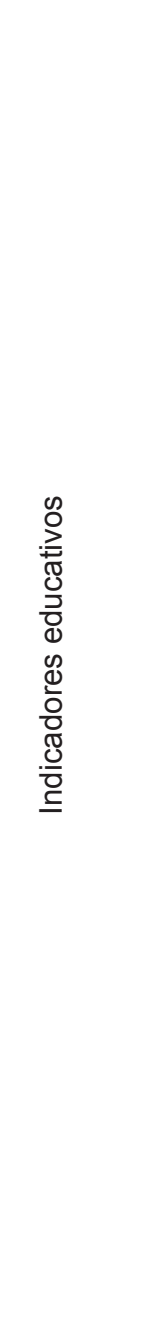 } & Matriculación & $\begin{array}{l}\text { En } 2016 \text {, en estudios de } \\
\text { grado se matricularon } \\
11847 \text { personas ( } 4702 \text { hom- } \\
\text { bres y } 7145 \text { mujeres). } \\
\text { En estudios de máster se } \\
\text { matricularon } 443 \\
\text { personas ( } 233 \text { hombres y } \\
210 \text { mujeres). } \\
\text { En estudios de doctorado } \\
\text { se matricularon } 14 \\
\text { personas (11 hombres y } \\
3 \text { mujeres). }\end{array}$ & $\begin{array}{c}\text { En 2016, en estudios de } \\
\text { grado se matricularon } 17425 \\
\text { personas (11641 hombres y } \\
5784 \text { mujeres). } \\
\text { En estudios de máster se } \\
\text { matricularon } 2594 \text { personas } \\
\text { (1734 hombres y } \\
860 \text { mujeres). } \\
\text { En estudios de doctorado o } \\
\text { equivalente, se matricularon } \\
25 \text { personas (19 hombres y } \\
6 \text { mujeres). }\end{array}$ & $\begin{array}{c}\text { En } 2016, \text { en estudios de } \\
\text { grado se matricularon } \\
673760 \text { personas ( } 357257 \\
\text { hombres y } \\
316503 \text { mujeres). } \\
\text { En estudios de máster } \\
\text { se matricularon } 94802 \\
\text { personas ( } 47392 \text { hombres } \\
\text { y } 47410 \text { mujeres). } \\
\text { En estudios de doctorado } \\
\text { se matricularon } 26906 \\
\text { personas (16629 hombres } \\
\text { y } 10277 \text { mujeres) } \\
\text { (UIS, } 2016 \mathrm{~d}) .\end{array}$ \\
\hline & $\begin{array}{l}\text { Tasas de } \\
\text { repetición y } \\
\text { deserción }\end{array}$ & & & \\
\hline & $\begin{array}{c}\text { Tasas de gra- } \\
\text { duación }\end{array}$ & $\begin{array}{l}\text { En } 2016 \text { se graduaron } 1289 \\
\text { personas en estudios de } \\
\text { grado o equivalente } \\
\text { (836 mujeres). } \\
\text { En estudios de máster o } \\
\text { equivalente, se graduaron } \\
18 \text { personas (7 mujeres). } \\
\text { En estudios de doctorado } \\
\text { no existen datos. }\end{array}$ & $\begin{array}{c}\text { En } 2016 \text { se graduaron } 2812 \\
\text { personas en estudios de gra- } \\
\text { do (934 mujeres). } \\
\text { En estudios de máster se } \\
\text { graduaron } 184 \text { personas } \\
\text { (47 mujeres) } \\
\text { En } 2015 \text { en estudios de } \\
\text { doctorado se graduaron } 9 \\
\text { personas ( } 1 \text { mujer). }\end{array}$ & $\begin{array}{c}\text { En } 2016 \text { se graduaron } \\
80270 \text { personas en estu- } \\
\text { dios de grado } \\
\text { (38751 mujeres). } \\
\text { En estudios de máster, se } \\
\text { graduaron } 21391 \text { personas } \\
\text { (10297 mujeres). } \\
\text { En estudios de doctorado } \\
\text { se graduaron } 1400 \text { perso- } \\
\text { nas (426 mujeres) } \\
\text { (UIS, } 2016 e) .\end{array}$ \\
\hline
\end{tabular}




\begin{tabular}{|c|c|c|c|c|}
\hline \multirow{5}{*}{ 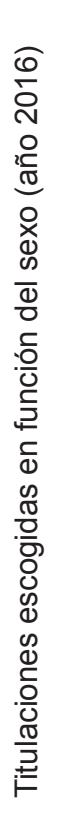 } & Educación & $\begin{array}{l}\text { El } 20 \% \text { de los graduados } \\
\text { eran hombres y el } 17 \% \\
\text { eran mujeres. }\end{array}$ & $\begin{array}{l}\text { El } 10 \% \text { de los graduados eran } \\
\text { hombres y el } 4 \% \text { mujeres. }\end{array}$ & $\begin{array}{c}\text { El } 5 \% \text { de los graduados } \\
\text { eran hombres y } \\
\text { el } 2 \% \text { mujeres. }\end{array}$ \\
\hline & Ciencias & $\begin{array}{l}\text { Un } 6 \% \text { de los graduados } \\
\text { eran mujeres y el } 21 \% \text { de } \\
\text { los graduados } \\
\text { eran hombres. }\end{array}$ & $\begin{array}{l}\text { El } 21 \% \text { eran hombres gradua- } \\
\text { dos y el } 16 \% \text { eran mujeres. }\end{array}$ & $\begin{array}{l}\text { E19\% de los graduados } \\
\text { eran hombres y } \\
\text { el } 17 \% \text { mujeres. }\end{array}$ \\
\hline & $\begin{array}{l}\text { Artes y Huma- } \\
\text { nidades }\end{array}$ & $\begin{array}{l}\text { El porcentaje de graduados } \\
\text { fue equivalente para } \\
\text { hombres y mujeres, un } 4 \% .\end{array}$ & $\begin{array}{c}\text { El } 20 \% \text { eran hombres y el } \\
19 \% \text { eran mujeres. }\end{array}$ & $\begin{array}{c}\text { Los porcentajes eran } \\
\text { semejantes para hombres y } \\
\text { mujeres, un } 14 \% \text {. }\end{array}$ \\
\hline & $\begin{array}{l}\text { Administración } \\
\text { de Empresas y } \\
\text { Derecho }\end{array}$ & $\begin{array}{c}\text { El } 33 \% \text { de los graduados } \\
\text { eran mujeres y el } \\
30 \% \text { eran hombres. }\end{array}$ & $\begin{array}{c}\text { El } 27 \% \text { eran hombres } \\
\text { graduados y el } \\
37 \% \text { eran mujeres. }\end{array}$ & $\begin{array}{c}\text { El } 18 \% \text { de los graduados } \\
\text { eran hombres y el } \\
21 \% \text { eran mujeres. }\end{array}$ \\
\hline & $\begin{array}{l}\text { Salud y } \\
\text { Bienestar }\end{array}$ & $\begin{array}{c}\text { El } 6 \% \text { de los graduados } \\
\text { eran hombres y el } 16 \% \text { eran } \\
\text { mujeres. }\end{array}$ & $\begin{array}{c}\text { El 0'54\% de los graduados } \\
\text { eran hombres y } 0 \text { ' } 3 \% \text { eran } \\
\text { mujeres. }\end{array}$ & $\begin{array}{l}\text { El 0'91\% de los graduados } \\
\text { eran hombres y el 1'66\% } \\
\text { eran mujeres (UIS, 2016c). }\end{array}$ \\
\hline \multirow{2}{*}{ 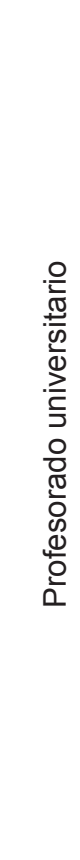 } & $\begin{array}{l}\text { Cantidad de } \\
\text { profesores y } \\
\text { profesoras } \\
\text { (año 2009) }\end{array}$ & $\begin{array}{c}\text { En } 2009 \text {, el número de } \\
\text { contratados eran } 177 \\
\text { profesores y } \\
121 \text { profesoras. } \\
\text { En } 2014 \text {, la cantidad de } \\
\text { contratados en Educación } \\
\text { Terciaria eran } 339 \\
\text { profesores y } 228 \text { profesoras. }\end{array}$ & $\begin{array}{c}\text { En } 2009 \text {, el número de contra- } \\
\text { tados eran } 345 \text { profesores y } \\
25 \text { profesoras. } \\
\text { En } 2014 \text {, El número de } \\
\text { contratados eran } 415 \\
\text { profesores y } 33 \text { profesoras. }\end{array}$ & $\begin{array}{c}\text { En } 2009 \text {, el número de } \\
\text { contratados eran } 11.970 \\
\text { profesores y } \\
3.400 \text { profesoras. } \\
\text { En } 2014 \text { no hay datos } \\
\text { (UIS, 2009) (UIS, 2014). }\end{array}$ \\
\hline & $\begin{array}{l}\text { Responsabili- } \\
\text { dades y cargos }\end{array}$ & $\begin{array}{c}\text { En la única universidad } \\
\text { pública de Cabo Verde, la } \\
\text { Universidad de Cabo Verde, } \\
\text { la rectora y vicerrectora del } \\
\text { Área de Extensión Universi- } \\
\text { taria son mujeres. Y hay un } \\
\text { hombre en el vicerrectorado } \\
\text { del Área de Integración } \\
\text { Tecnológica e innovación } \\
\text { (UNICV, s.f.). }\end{array}$ & $\begin{array}{l}\text { En la única universidad } \\
\text { pública de Mauritania, La } \\
\text { universidad de Nouakchott } \\
\text { el presidente es un hombre y } \\
\text { por lo tanto no hay mujeres en } \\
\text { los altos cargos (UNM, s.f.). }\end{array}$ & $\begin{array}{c}\text { De las trece universidades } \\
\text { públicas que hay, se puede } \\
\text { observar que estas univer- } \\
\text { sidades están presididas } \\
\text { por hombres y por lo tanto, } \\
\text { no hay mujeres } \\
\text { (UAE, s.f.) (UCA, s.f.) } \\
\text { (UCD, s.f.) (UH1, s.f.) } \\
\text { (UNIVH2, s.f.) (UIZ, s.f.) } \\
\text { (UMP, s.f.) (USMS, s.f.) } \\
\text { (USMBA, s.f.). }\end{array}$ \\
\hline
\end{tabular}

\section{DISCUSIÓN Y CONCLUSIONES}

A partir de las debilidades detectadas se han establecido una serie de propuestas de mejora tanto a nivel general como específico para cada país. En cuanto a nivel general:

- Marruecos y Mauritania podrían tomar como referencia el Instituto Caboverdiano para la Igualdad y Equidad de Género de Cabo Verde, donde se publican datos e informes sobre la situación de la mujer a nivel general y educativo. Esto le permite al país ver los avances en materia de género y tomar las medidas necesarias para empoderar a la mujer caboverdiana.

- Promover becas para cursar estudios de máster y doctorado, ya una de las causas que impiden a las mujeres cursar este tipo de estudio son cuestiones económicas.

- Las universidades podrían llevar a cabo campañas de sensibilización (tanto en el ámbito universitario como en la sociedad en general) que empoderen la figura de la mujer en todos los aspectos de la vida social, la investigación, el crecimiento económico... Además de programas de prevención de matrimonios y embarazos no deseados. 
- Incluir contenidos relacionados con la igualdad y violencia de género en todas las titulaciones y formación específica al profesorado.

- Las universidades podrían realizar estudios anuales sobre las tasas de repetición y abandono con el objetivo de establecer sus propias medidas para evitarlo.

- Promover el acceso de las mujeres a todas las titulaciones, así como su contratación como docentes e investigadoras en todos los campos académicos. De esta manera podría existir una paridad de género en la Educación Superior y aumentaría el número de profesoras y alumnas en las universidades.

- Concesión de ayudas para el acceso a la Educación Superior para aquellas mujeres situadas en espacio rurales. A través de estas becas podrían pagarse sus estudios así como su estancia fuera del hogar y evitar su temprana incorporación en trabajos precarios.

- Creación de políticas que faciliten la conciliación familiar, para que la mujer pueda combinar trabajo-estudio-familia. Gracias a estas políticas se podría evitar la no contratación de las mismas por el hecho de ser madres.

Las universidades deben de tener un protocolo para la detección, prevención y actuación en los supuestos de acoso sexual y sexista, con el objetivo de garantizar la seguridad de las alumnas, profesoras e investigadoras en los espacios universitarios.

Por otra parte, en cuanto a las propuestas específicas para cada país:

\section{Cabo Verde}

- Fomentar políticas de empleo para la inserción laboral de las mujeres: establecimiento de convenios entre las universidades y las instituciones. Las universidades pueden establecer convenios con dichas instituciones para que las prácticas externas se convirtieran en un periodo de adaptación y aprendizaje. Y si ha adquirido las habilidades y competencias, poder obtener el puesto de trabajo.

- Implementar políticas de becas dirigidas a mujeres para fomentar su acceso a los estudios de máster y doctorado. A través de estas becas aumentaría la cantidad de mujeres profesoras y el número de alumnas en las universidades.

- Existencia de un evaluador externo en las universidades. A través de este evaluador se podría garantizar que las medidas establecidas se están cumpliendo, conocer si sigues existiendo casos de desigualdad, etc.

\section{Mauritania}

- Implementar políticas de becas para fomentar el acceso de la mujer a estudios superiores. Uno de los mayores problemas en Marruecos son los matrimonios de chicas jóvenes con hombres por dinero sobre todo entre familias pobres y de zonas rurales, para que sus familias puedan vivir y esto dificulta su acceso a la educación. Por ello, si se ofrecieran ayudas estas podrían estudiar sin que sus padres se preocuparan de los pagos.

\section{Marruecos}

- Establecer medidas concretas para fomentar la inclusión de la mujer en la educación superior, ya que en Marruecos se han llevado a cabo programas dirigidos a evitar la discriminación de la mujer en las instituciones educativas, pero la mayoría se orientan a enseñanza primaria o secundaria. Además, para valorar el cumplimiento de estas medidas, se propone la existencia de un evaluador externo.

Como conclusión, es importante destacar que la desigualdad de género seguirá existiendo si no cambia el pensamiento y la cultura tradicional de la sociedad marroquí, mauritana y caboverdiana. Por ello, llevar a cabo políticas de igualdad es el primer paso necesario para que se produzcan cambios significativos en un futuro. No obstante, es totalmente necesario que los gobiernos y los ministerios de educación de cada país se comprometan con la inclusión de la mujer en la educación en todos los niveles educativos, porque si algo está demostrado es que la educación de las mujeres tiene un impacto significativo en su concepción sobre sí mismas, sobre sus prioridades y su futuro. Son las mujeres las que cambian el mundo, son las mujeres las que logran la igualdad de género y son las mujeres caboverdianas, mauritanas y marroquís las que tienen el poder de cambiar sus sociedades. Pero para ello, necesitan contar con el apoyo de Gobiernos y los Ministerios de Educación para que nada ni nadie les impida formarse. 


\section{REFERENCIAS BIBLIOGRÁFICAS}

Banco Mundial (2012). Construindo Futuro: Como é que o Ensino Superior Pode Contribuir Para a Agenda de Transformação Económica e Social de Cabo Verde [PDF]. Recuperado de: https://www.dgesc.gov.cv/index.php/ensino-superior-de-cv/ estudos-sobre-es-de-cv/send/10-estudos-sobre-es-em-cv/1-estudo-sobre-ensino-superior-cabo-verdiano.

CNDH (2015). Balance sobre la igualdad y la paridad en Marruecos. Resumen ejecutivo [PDF]. Rabat, CNDH. Recuperado de: http://cndh.ma/sites/default/files/cndh_-_r.e_-_web_parite_egalite_es_-.pdf.

Instituto de Estadística de la UNESCO (UIS) (2014): Docentes por nivel de enseñanza. Educación Terciaria. Instituciones públicas. Recuperado de: http://uis.unesco.org/indicator/edu-hr-teach-level.

Instituto de Estadística de la UNESCO (UIS) (2009): Docentes por nivel de enseñanza. Educación Terciaria. Instituciones públicas. Recuperado de: http://uis.unesco.org/indicator/edu-hr-teach-level.

Instituto de Estadística de la UNESCO (UIS) (2016a). Enrolment by level education [Base de datos]. Recuperado de: http:// data.uis.unesco.org/index.aspx?queryid=147\&lang=en.

Instituto de Estadística de la UNESCO (UIS) (2016b). Tertiary graduates by level education. [Base de datos]. Recuperado de: http://data.uis.unesco.org/index.aspx?queryid=147\&lang=en.

Instituto de Estadística de la UNESCO (UIS) (2016c): Distribución de graduados de terciaria por campo de educación (hombre y mujer): Educación; Ciencias; Administración de Empresas y Derecho; Artes y Humanidades; y Salud y Servicios. Recuperado de: http://uis.unesco.org/indicator/edu-compl-grad-field.

Instituto de Estadística de la UNESCO (UIS) (2016d). Enrolment by level education [Base de datos]. Recuperado de: http:// data. uis. unesco.org/index.aspx?queryid=147\&lang=en.

Instituto de Estadística de la UNESCO (UIS) (2016e). Tertiary graduates by level education. [Base de datos]. Recuperado de: http://data.uis.unesco.org/index.aspx?queryid=147\&lang=en.

Inter Press Service (IPS) (s.f.): Unión Africana: ante todo los derechos de la mujer. Recuperado de: http://www.ipsnoticias. net/2016/02/union-africana-ante-todo-los-derechos-de-la-mujer/.

Lopes da Silva, D. (2016a): A Feminização da Pobreza em Cabo Verde. Instituto caboverdiano para la Igualdades e Equidade de Género (ICIEG) [PDF]. Recuperado de: http://ine.cv/ObservatorioGenero/index.php/artigos-e-relatorios/ send/2-artigos/39-a-feminizacao-da-pobreza-em-cabo-verde-draft1.

Lopes da Silva, D. (2016b): O acesso a educação e autonomia económica. Instituto caboverdiano para la Igualdades e Equidade de Género (ICIEG) [PDF]. Recuperado de: http://ine.cv/ObservatorioGenero/index.php/artigos-e-relatorios/ send/2-artigos/41-o-acesso-a-educacao-e-autonomia-economica-dr-draft1.

UNESCO (2017a). Analyse de la situationde la violence en milieu scolaire au maroc. Rabat, UNESCO [PDF]. Recuperado de: http://www.unesco.org/new/fileadmin/MULTIMEDIA/FIELD/Rabat/images/ED/20171219AnalyseStituationMaroc.PDF.

Universidad Abdelmalek Essaâdi (UAE) (s.f.): Equipe de la présidence. Recuperado de: https://www.uae.ma/website/fr/universit\%C3\%A9/equipe-pr\%C3\%A9sidence.

Universidad Cadi Ayyad (UCA) (s.f.): Mont du président. Recuperado de: https://www.uca.ma/.

Universidad Chouaib Doukkali (UCD) (s.f.): La présidence. Recuperado de: http://www.ucd.ac.ma/index.php?option=com_ k2\&view=item\&layout=item\&id=242\&ltemid=768.

Universidad de Cabo Verde (UNICV) (s.f.): Equipa reitoral. Recuperado de: http://www.unicv.edu.cv/apresentacao/reitoria.

Universidad Hassan I (UH1) (s.f.): Gouvernance. Recuperado de: http://www.uh1.ac.ma/.

Universidad Hassan II Mohammedia (UNIVH2) (s.f.): Gouvernance. Recuperado de: http://www.univh2c.ma/d_page.aspx?mgr=14.

Universidad Ibn Zohr (UIZ) (s.f.): Présidence. Recuperado de: http://www.uiz.ac.ma/index.php/presidence.

Universidad Mohammed Pemier Oujda (UMP) (s.f.): Présidence. Recuperado de: http://www.ump.ma/fr/luniversite/presidence. Universidad Moulay Slimane (USMS) (s.f.): Présidence. Recuperado de: http://usms.ac.ma/?page_id=3716.

Universidad Sidi Mohamed Ben Abdellah (USMBA) (s.f.): Gouvernance. Recuperado de: http://www.usmba.ac.ma/ usmba2/. 